\title{
Adaptive Electronic Dispersion Compensator for Chromatic and Polarization-Mode Dispersions in Optical Communication Systems
}

\author{
Ut-Va Koc \\ Bell Labs, Lucent Technologies, 600 Mountain Avenue, Murray Hill, NJ 07974, USA \\ Email: koc@bell-labs.com
}

Received 1 April 2004; Revised 30 November 2004

\begin{abstract}
The widely-used LMS algorithm for coefficient updates in adaptive (feedforward/decision-feedback) equalizers is found to be suboptimal for ASE-dominant systems but various coefficient-dithering approaches suffer from slow adaptation rate without guarantee of convergence. In view of the non-Gaussian nature of optical noise after the square-law optoelectronic conversion, we propose to apply the higher-order least-mean $2 \mathrm{~N}$ th-order (LMN) algorithms resulting in OSNR penalty which is $1.5-2 \mathrm{~dB}$ less than that of LMS. Furthermore, combined with adjustable slicer threshold control, the proposed equalizer structures are demonstrated through extensive Monte Carlo simulations to achieve better performance.
\end{abstract}

Keywords and phrases: electronic PMD compensation, adaptive equalization, signal processing, optical communication, leastmean fourth-order algorithm, least-mean-square algorithm.

\section{INTRODUCTION}

Optical communication forms the backbone of modern telecom and Internet networks around the globe. Due to its enormous inherent channel capacity [1], it is anticipated that this trend will continue or even accelerate. In this ongoing evolution, adaptive electronic equalization for combating impairments in fiber-optic communication may play an important role in pushing from the core of networks all the way to the edge by providing cost-effective solution. Two major impairments commonly encountered in modern fiberoptic systems are chromatic dispersion (group velocity dispersion or GVD) and polarization-mode dispersion (PMD). Chromatic dispersion can be compensated effectively by an optical dispersion compensation module (DCM) due to its static nature. However, at substantially high data rates (10 or even $40 \mathrm{Gbps}$ ), especially in long-haul networks, residual chromatic dispersion amount remains problematic and thus electronic equalization against residual chromatic dispersion is still important [2]. In cost-sensitive metro networks, electronic solution is considered a viable option to replace the expensive optical solution. On the other hand, PMD is dynamic in nature and substantial unpredictable PMD is accumulated over a long distance of old fibers, enough to cause network outage [3]. Currently it is extremely expensive to be compensated optically by bulky optical PMD compensators (OPMDCs) and thus electronic solution is vigorously sought in recent years.
Adaptive electronic equalizers for impairment compensation in fiber-optic networks have been studied for decades. In early work [4], the dominant noise was quantum, shot, or electronic thermal noise, which can be modeled effectively as additive Gaussian noise. After the advent of efficient and low-noise fiber amplifiers in 1987 [5], optical amplifiers (EDFA or Raman) were used extensively to increase the transmission distance without O-E-O conversion. Since then, a number of studies were undertaken to explore a variety of equalizer structures for adaptive optical-channel impairment compensation ranging from feedforward-type equalizers to maximum-likelihood estimators $[6,7,8,9$, $10,11]$. Among many studies in electronic PMD compensation in recent years, $[12,13,14,15,16,17,18,19]$, the first decision-feedback loop and tapped-delay-line equalizer at $10 \mathrm{Gbps}$ were implemented in [20] and [21] respectively. The maximum-likelihood estimation for PMD compensation was also investigated in $[22,23]$.

In a fiber-optic link, a number of optical amplifiers, either erbium-doped fiber amplifiers (EDFAs) or Raman amplifiers (RAs), are employed to strengthen the optical signal, but at the same time add in the incoherent amplified spontaneous emission (ASE) noise (commonly called optical noise) [5]. In the quantum regime, the ASE noise follows the Bose-Einstein distribution [24] and the optically amplified coherent light exhibits a noncentral-negative-binomial distribution [25]. However, in the classical limit when the signal and noise involve many photons, this optical noise can be 


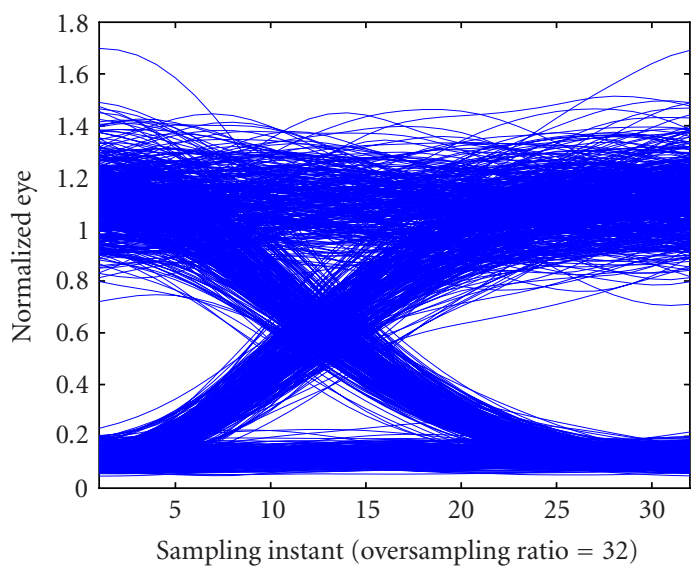

(a)

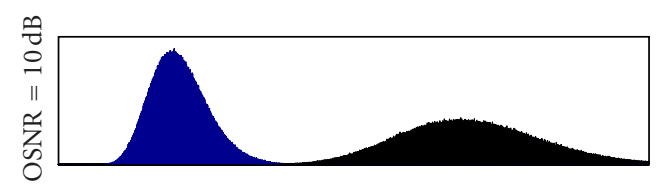

(c)

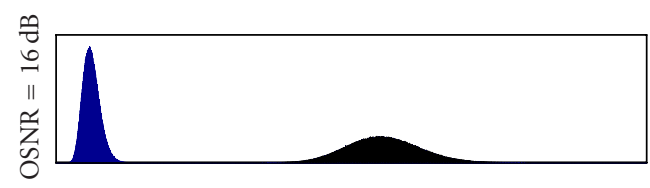

(e)

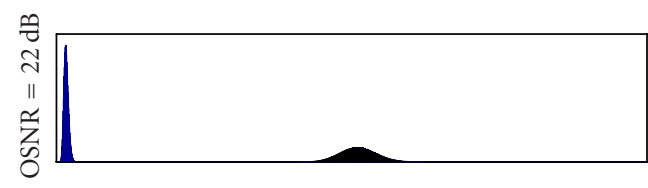

(g)

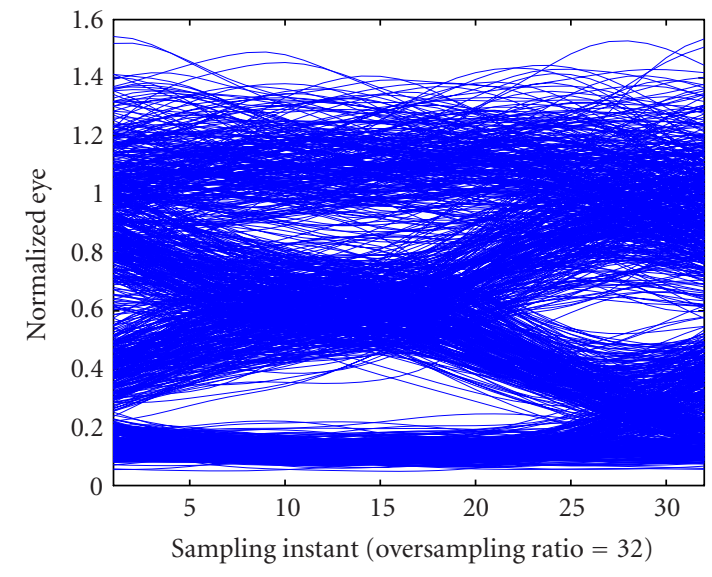

(b)

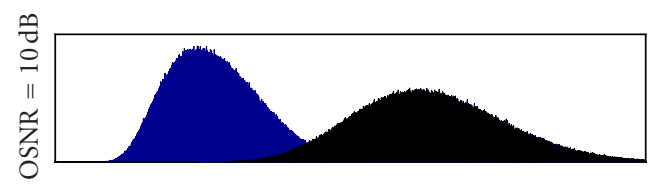

(d)

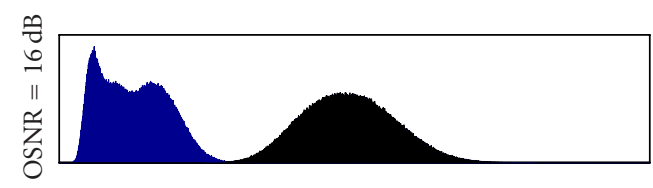

(f)

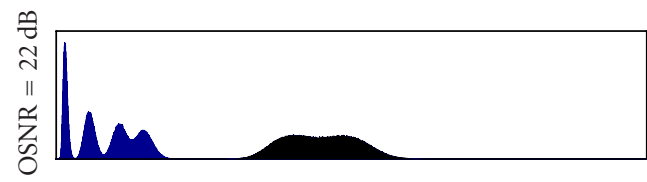

(h)

FIGURE 1: (a) and (b) Eye diagrams when OSNR = $16 \mathrm{~dB}$ and (c), (d), (e), (f), (g), and (h) histograms of noise distribution of bits 1 and 0 at the decision point for NRZ when OSNR is equal to (c), (d) $10 \mathrm{~dB}$; (e), (f) $16 \mathrm{~dB}$; and (g), (h) $22 \mathrm{~dB}$. (Left column: DGB=0 picoseconds; Right column: DGB = 75 picoseconds; both are at 10 Gbps NRZ.)

modeled as complex additive white Gaussian noise (AWGN) in the optical field of each orthogonal polarization mode of the optically amplified signal based on the central limit theorem $[26,27]$. An optical filter $H_{o}(f)$ is usually placed before a photodetector to limit the optical noise reaching the receiver and also to filter out other unselected channels in a wavelength-division multiplexing (WDM) system.

At the receiver side, after square-law detection using a photodiode, the noise becomes non-Gaussian. As a result, the noisy input signal $u(t)$ contains a signal-dependent nonsymmetric Gaussian term having a variance determined by the signal levels of bits ( 0 and 1$)$, and a nonzero-mean noncentral $\chi^{2}$ term. It should be noted that the mean of $u(t)$ is nonzero and varies at different optical signal-to-noise ratio levels (OSNR), as depicted in Figures $1 \mathrm{a}$ and $1 \mathrm{~b}$ without
PMD (i.e. differential group delay or DGD $=0$ picosecond) and with substantial amount of PMD (DGD $=75$ picoseconds), respectively, where the eye diagram and noise distributions of bits 0 and 1 at the decision point for NRZ at different OSNR levels are plotted to demonstrate the varying nonzero mean values. This noise property is very different from electronic thermal noise or even optical noise after the combination of an interferometer and a balanced receiver [28].

Because of this non-Gaussian noise property, the wellstudied least-mean-square (LMS) algorithm becomes suboptimal for optical noise in the sense that OSNR penalty is observed even without the presence of PMD-induced intersymbol interference (ISI), though it is optimal for additive Gaussian noise [29]. Various coefficient-dithering approaches 
were devised to replace LMS but generally suffer from slow adaptation rate and no guarantee of convergence. Typically, tap coefficients are adjusted in a trial-and-error manner with feedback from various types of error monitors such as minimization of eye-monitoring pseudoerror count [30], maximization of eye-opening [31], Q-factor optimization [32], bit-pattern-dependent threshold control [9], or FEC-errorcount minimization [33].

The higher-order statistic techniques, the least-mean $2 \mathrm{~N}$ th-order (LMN) family, were first proposed in [34] as an extension to the LMS and were found better suited than the LMS for non-Gaussian noise [34, 35]. In this paper, we propose to apply these higher-order statistic algorithms to coefficient update for combating the non-Gaussian optical noise after photodetection. Through Monte Carlo simulation, we demonstrate that $1.5-2 \mathrm{~dB}$ better OSNR penalty can be attained for the LMN than the LMS [36]. Furthermore, due to the nonsymmetric nature of optical noise, we also propose to combine these higher-order algorithms with automatic slicer threshold control (ATC-8LICER) and show that the threshold-optimized LMN still achieves better results than the conventional LMS with optimized thresholds. In Section 2, we briefly introduce the higher-order algorithms (LMN) in comparison to the LMS algorithm for feedforward (FFE) and decision-feedback (DFE) equalizers. In Section 3, the results through extensive Monte Carlo simulation are presented to evaluate the performance of the LMN algorithms combined with automatic threshold control compared against the LMS. Finally, the conclusion is drawn in Section 4.

\section{ARCHITECTURES AND ALGORITHMS OF ELECTRONIC PMD COMPENSATORS}

In a digital receiver architecture, an analog-to-digital converter (ADC) is required to digitize the incoming bandlimited analog signal for further processing in the digital adaptive equalizer. Though more complex functionality can be achieved in low-power CMOS digital circuitry coupled with pipelined/parallel data flows after digitization, the ADC is considered one of the bottlenecks for digital implementation of advanced equalizers. The current state-of-the-art ADC can provide about 6-bit accuracy at $10 \mathrm{Gbps}$ but it becomes challenging at $40 \mathrm{Gbps}$. Furthermore, the ADC power consumption at such a high speed may offset the low-power benefit of digital CMOS circuits. On the other hand, the analog equalizer, particularly the simple analog tapped-delay-line equalizer, is an attractive alternative in the absence of ADC for providing a practical electronic dispersion compensation solution. No matter what implementation (digital or analog) is adopted, the operations of an equalizer can be described in the discrete-time or $z$-domain. In this paper, we express equalizer algorithms in the discrete-time domain without explicitly referring to a digital or analog implementation.

A number of electronic equalizer structures, such as feedforward equalizer (FFE), decision-feedback equalizer (DFE), and maximum-likelihood sequence estimator (MLSE), have been considered over the years. Though MLSE achieves the best performance, substantial complexity and compromises are often associated with practical implementation. Specificially, MLSE has exponential complexity $O\left(e^{N}\right)$ but FFE/DFE requires only linear complexity $O(N)$. However, the best reason for using relatively simple architectures (FFE/DFE) in most wireless/wireline electronic systems instead of MLSE is that they usually offer entirely adequate performance [37]. In this paper, we examine two basic equalizer architectures: feedforward equalizers (FFEs) and decision-feedback equalizers (DFEs) with FFE, as shown in Figures $2 a$ and 2b, respectively.

\subsection{Least-mean $2 N$ th-order algorithms}

It has been shown that the LMS algorithm is $H^{\infty}$-optimal under the assumption of temporal whiteness and Gaussian disturbances [38], providing theoretical justification for the excellent robustness of LMS. However, after square-law detection by a photodiode, optical noise becomes nonzero-mean signal-dependent $\chi^{2}$. As a result, the conventional LMS algorithm becomes suboptimal for optical noise in the sense that equalization introduces OSNR penalty even without any ISI [29], though it is optimal for additive Gaussian noise (e.g. thermal noise). The higher-order statistic techniques, the least-mean $2 N$ th-order (LMN) family, were proposed in [34] as a higher-order extension of LMS and found to be better suited for non-Gaussian noise than LMS [35]. Due to the non-Gaussian nature of optical noise, we propose to employ the higher-order counterparts of LMS which are found to yield better results than LMS.

After equalization, a slicer makes a hard decision to determine the estimated symbol $\hat{d}(k)$ (or bit in the OOK case) from the compensated slicer input $s(k)$ in reference to a slicer threshold. For the case of only two signal levels, the slicer is simply a high-speed comparator as used in a conventional CDR. The LMS minimizes the cost function of the square of the slicer error $e(k): J_{\mathrm{LMS}}(k)=E\left\{e^{2}(k)\right\}$, where $e(k)=a(k)-s(k)$ is the difference between the target signal $a(k)$ and the equalized signal $s(k)$ before decision. In the normal mode, $a(k)=\hat{d}(k)$ but, in the training mode, $a(k)=d(k)$, the transmitter input bit. Usually a training sequence is required at the startup stage of equalization and then the equalizer is switched to the normal mode. However, it is possible to start the equalizer without any training sequence through blind startup which is out of the scope of this paper. In fiber-optic communication, fixed bytes such as SONET A1/A2 bytes are sent in every frame and can be utilized as the training sequence. As an extension of the LMS, the LMN is a class of adaptive algorithms to minimize the cost function of the $2 \mathrm{Nth}$ order of $e(k) J(k)=E\left\{e^{2 N}(k)\right\}$. For $N=1$, the LMN algorithm becomes LMS. For $N=2$, it is called the least-mean fourthorder (LMF) algorithm. When $N=3$, it is the least-mean sixth-order (LM6) algorithm. Since the LMS belongs to the LMN family, the extensive knowledge of the LMS in both theory and implementation can also be applied to the LMN family. 


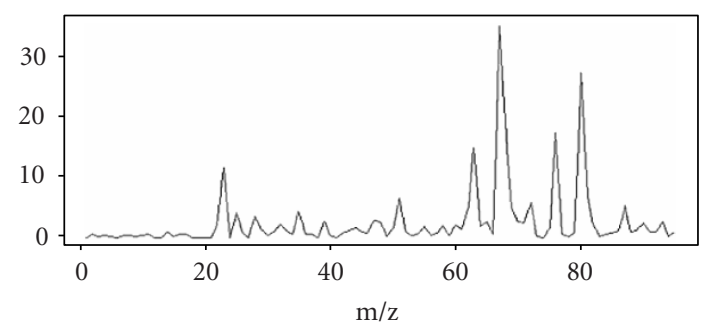

(a)

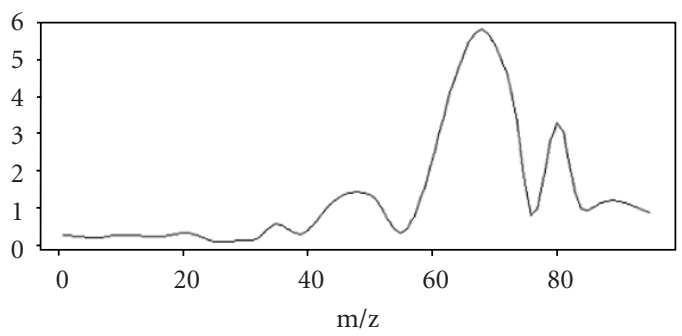

(b)

FIgURE 2: Different equalizer architectures are considered. The filter response of FFE is $C(z)$ while the response of DFE is $B(z)$. The decision level is fixed to the midpoint of the bit values $\{0,1\}$. (a) Feedforward equalizer (FFE). (b)Decision feedback equalizer (DFE) coupled with an FFE.

Because the first and second moments (equivalently mean and variance) are sufficient to describe Gaussian distributions, it is not necessary to account for higher moments in the cost function and the second-order cost function in the LMS is enough for Gaussian noise. For non-Gaussian noise, higher moments are required. Intuitively, the higher-order cost function wages a large penalty for a large slicer error to account for higher moments and causes the noise distribution to be more compact for non-Gaussian noise, as will be seen in Section 3.

\subsection{Feedforward equalizers}

Consider the case of FFE depicted in Figure 2a where the $(2 L+1)$-tap FFE coefficients are $\left\{c_{-L}(k), \ldots, c_{L}(k)\right\}$ at $t=k T_{\mathrm{s}}$ in the discrete-time domain. Here $T_{\mathrm{s}}=1 / f_{\mathrm{s}}$ is the sample period. If the oversampling ratio $R=T / T_{\mathrm{s}}>1$, then the FFE is called a fractionally-spaced equalizer. In vector form, these weights can be denoted as $\overrightarrow{\mathbf{c}}^{T}(k)=\left[c_{-L}(k), \ldots, c_{L}(k)\right]$. The receiver input data sequence is $\overrightarrow{\mathbf{u}}^{T}(k)=[u(k+L), \ldots, u(k-$ $L)]$. Therefore, the slicer input is $s(k)=x(k)=\overrightarrow{\mathbf{c}}^{T}(k) \overrightarrow{\mathbf{u}}(k)$. The weight updating unit of $C(z)$ (WUD $[C]$ ) adapts $\overrightarrow{\mathbf{c}}(k)$ based on an adaptive algorithm, among which the LMS is the most widely used. As in Figure 2a, the weight updating unit $C(z)$ adopts the $\mathrm{LMN}$ of $N$ th order. It can be shown that

$$
\begin{aligned}
\overrightarrow{\mathbf{c}}(k+1) & =\overrightarrow{\mathbf{c}}(k)-\frac{\beta}{2} \nabla_{C}\left\{[e(k)]^{2 N}\right\} \\
& =\overrightarrow{\mathbf{c}}(k)+\beta N[e(k)]^{2 N-1} \overrightarrow{\mathbf{u}}(k),
\end{aligned}
$$

where $\beta$ is the preset step size.

\subsection{Decision-feedback equalizers with FFE}

For the case of a decision-feedback equalizer (DFE) coupled with an FFE, as in Figure 2b, where $s(k)=x(k)-y(k)=$ $\overrightarrow{\mathbf{c}}^{T}(k) \overrightarrow{\mathbf{u}}(k)-\overrightarrow{\mathbf{b}}^{T}(k) \overrightarrow{\mathbf{a}}(k)$ with the DFE coefficients defined as $\overrightarrow{\mathbf{b}}^{T}(k)=\left[b_{1}(k), \ldots, b_{M}(k)\right]$. Here $\overrightarrow{\mathbf{a}}^{T}(k)=[a(k-1), \ldots, a(k-$ $M)]$. The weight updating units for FFE and DFE are denoted as $\operatorname{WUD}(C)$ and $\operatorname{WUD}(B)$, respectively and can employ a variety of adaptive algorithms including the LMN. In Figure $2 \mathrm{~b}, s(k)=x(k)-y(k)=\overrightarrow{\mathbf{c}}^{T}(k) \overrightarrow{\mathbf{u}}(k)-\overrightarrow{\mathbf{b}}^{T}(k) \overrightarrow{\mathbf{a}}(k)$. Define $\overrightarrow{\mathbf{w}}^{T}(k)=[\overrightarrow{\mathbf{c}}(k),-\overrightarrow{\mathbf{b}}(k)]$ and $\overrightarrow{\mathbf{r}}^{T}(k)=[\overrightarrow{\mathbf{u}}(k),-\overrightarrow{\mathbf{a}}(k)]$, where $\overrightarrow{\mathbf{c}}^{T}(k)=\left[c_{-L}(k), \ldots, c_{L}(k)\right], \overrightarrow{\mathbf{u}}^{T}(k)=[u(k+L), \ldots, u(k-L)]$, $\overrightarrow{\mathbf{b}}^{T}(k)=\left[b_{1}(k), \ldots, b_{M}(k)\right], \overrightarrow{\mathbf{a}}^{T}(k)=[a(k-1), \ldots, a(k-M)]$. We can rewrite $e(k)=a(k)-\overrightarrow{\mathbf{w}}^{T}(k) \overrightarrow{\mathbf{r}}(k)$. Therefore, the coefficients of the FFE and DFE can be updated as follows to minimize the cost function, $J(k)=E\left\{[e(k)]^{2 N}\right\}$ :

$$
\begin{aligned}
\overrightarrow{\mathbf{w}}(k+1) & =\overrightarrow{\mathbf{w}}(k)-\frac{\beta}{2} \nabla_{w}\left\{[e(k)]^{2 N}\right\} \\
& =\overrightarrow{\mathbf{w}}(k)+\beta N[e(k)]^{2 N-1} \overrightarrow{\mathbf{r}}(k) .
\end{aligned}
$$

For the first-order PMD channel impulse response $h(t)=$ $\gamma \delta(t)+(1-\gamma) \delta\left(t-\tau_{\mathrm{DGD}}\right)$, it is easy to show that there exist dips in the frequency response at locations inversely proportional to $\tau_{\mathrm{DGD}}$, especially for $\gamma=0.5$ [19]. It has been known that an FFE amplifies the noise in the spectral dips whereas a DFE does not [37]. Thus, the performance of FFE for PMD compensation in the high-DGD region is anticipated to be less than that of DFE.

\subsection{Slicer with automatic threshold control}

In the uncompensated OOK case, the two noiseless signal levels for bits 0 and 1 are normalized to 0 and 1 with the midpoint defined at 0.5 (or equivalently to $\{-1,1\}$ with the midpoint at 0 ). In view of the nonsymmetric distributions for bits 0 and 1 (having different variances) reflected in the signal-dependent term after square-law detection, the optimal threshold point is usually not the midpoint. Therefore, an automatic threshold control algorithm (ATC) is designed to track the incoming signal profile and automatically adjust the threshold in the CDR for the minimum bit error rate [39]. In this paper, the ideal ATC algorithm is employed by searching the entire simulation sequence for the best threshold value to obtain the least number of errored bits. In a practical system, the ATC algorithm usually accumulates signal distribution information within a window of finite duration for optimal threshold calculation to allow tracking of slowly varying nonstationary channels [40].

In the conventional equalizer structures illustrated in Figures $2 \mathrm{a}$ and $2 \mathrm{~b}$, the decision point (slicer threshold $\theta$ ) is fixed at the midpoint of two noiseless signal levels. Similar to the uncompensated case, the slicer input distributions for bits 0 and 1 are also nonsymmetric and thus result in the optimal threshold point being away from the midpoint. Therefore, we propose alternative architectures for FFE/DFE with adjustable threshold control in the slicer (ATC-SLICER) as shown in Figures 3a and 3b. The ATC-SLICER's function is to 


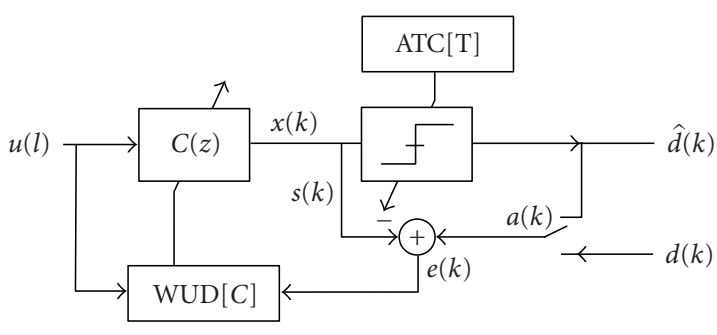

(a)

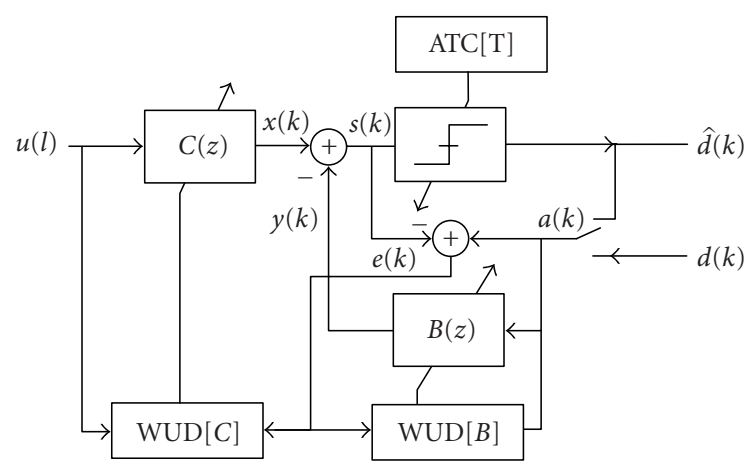

(b)

FIgure 3: Equalizers with automatic threshold adjustment (ATCSLICER). The decision threshold is controlled adaptively by an automatic threshold control (ATC) algorithm instead of being fixed at the midpoint of two noiseless signal levels. (a) FFE with ATCSLICER. (b) DFE with FFE and ATC-SLICER.

track the slicer input histogram and adjust the slicer threshold accordingly. In the steady state of equalization, the ATCSLICER can start to build up a slicer input profile to determine the best threshold for the slicer, and then dynamically track and adjust the optimal slicer threshold.

There are many ways readily available in the literature to implement the automatic threshold control technique (ATC). In our case of adaptive equalization, the input to the ATC-SLICER control block is the slicer input $s(k)$. In one of many possible implementations, a histogram is established in two arrays of memory, $\operatorname{bin}_{1}(i)$ and $\operatorname{bin}_{0}(i)$, for $i=0, \ldots, B+1$ corresponding to the intervals $\left\{\left(-\infty, v_{L}\right),\left[v_{L}, v_{L}+d\right),\left[v_{L}+\right.\right.$ $\left.\left.d, v_{L}+2 d\right), \ldots,\left[v_{L}+(i-1) d, v_{L}+i d\right), \ldots,\left[v_{H}-d, v_{H}\right],\left(v_{H}, \infty\right)\right\}$, where $d=\left(v_{H}-v_{L}\right) / B$ and $B$ is the number of bins that form the range from $v_{L}$ to $v_{H}$. The value of $B$ dictates the accuracy of the final threshold determination but a large $B$ value requires more memory space. In general, $B$ could be 128, 256, or even 1024. If $s(k)$ is in the interval of $\left[v_{L}+(i-1) d, v_{L}+i d\right)$, then the count in the memory $\operatorname{bin}_{1}(i)$ is incremented by one for the slicer output being 1 and $\operatorname{bin}_{0}(i)$ is incremented for the slicer output being 0 . The bit error can be estimated as follows: error[0] $=\operatorname{bin}_{1}[0]-\operatorname{bin}_{0}[0]$, and error $[i]=$ error $[i-1]+\operatorname{bin}_{1}[i]-\operatorname{bin}_{0}[i]$ for $i=1, \ldots, B+1$. The optimal threshold is determined by finding the minimum of error $[i]$. To avoid the detrimental memory effect for a changing optical channel, a reset signal is sent periodically to clear the memory arrays and a histogram is rebuilt fresh in accordance with the above rule. Since PMD drifts very slowly, it is possible to refresh this profile once one or few minutes without encountering significant change in channel response while the number of bits collected in that time frame is large enough for meaningful profiling. For an analog implementation of the equalizer, the compensated signal can be sampled at a low speed to build the profile of $s(k)$ over time. The optimal threshold can be determined externally through the method described above.

\section{RESULTS AND DISCUSSION}

An extensive Monte Carlo simulation is conducted in NRZ and RZ formats at $10 \mathrm{Gbps}$ to compare the performance of the LMN algorithms with/without automatic threshold control against the common LMS algorithm. The full-width half-maximum (FWHM) of the RZ pulse is 50\%. The simulation length is chosen dynamically at each OSNR level with a given amount of DGD to provide sufficient samples for reliable BER estimation. It usually requires a longer simulation for an expected low BER value in a given OSNR-DGD condition, but a shorter one for an expected high BER. The ATC algorithm finds the optimum threshold value on the uncompensated electronic signal over the entire simulation sequence. Its performance curves provide the benchmark, against which the performance charts of all the equalizer structures are compared. In order to isolate the effect of compensation on other impairments such as poor extinction ratio (ER), we deliberately set $\mathrm{ER}=30 \mathrm{~dB}$ and use a wideband optical bandpass filter for the simulation. The electronic lowpass filter is set to be a third-order Bessel filter of bandwidth $7 \mathrm{GHz}$ for $10 \mathrm{Gbps}$, whereas the optical filter is modeled as a tenth-order Gaussian filter with a bandwidth of $150 \mathrm{GHz}$ to weakly filter any receiving signal without causing any distortion. The peak optical power of the transmitter laser is controlled at $19.95 \mathrm{~mW}$ to avoid any nonlinear distortion during transmission, though no fiber nonlinearity is modeled in the interest of fast simulation. Only the first-order PMD and GVD are considered in the simulation. The first-order PMD is commonly characterized by the amount of DGD in picoseconds and the first-order GVD is characterized by the amount of dispersion in picoseconds per a nanometer of wavelength (ps/nm) without explicitly specifying the actual fiber type and length.

For the sake of brevity, we pick a fractionally spaced FFE of 9 taps at an oversampling ratio 2 coupled with a DFE of 2 feedback taps as the typical equalizer architecture (designated as FFE9 + DFE2) to illustrate the advantage of the higher-order LMN algorithms over the LMS, even though we have simulated a large variety of equalizer structures. The OSNR penalty curves of this equalizer are plotted in Figure 4 with and without ATC-SLICER (for optimized and fixed thresholds). The dash lines represents the use of the LMS, and the solid lines for the LMF. These OSNR penalty curves are aggregated from the OSNR-BER plots, of which two examples are shown in Figures $5 \mathrm{a}$ and $5 \mathrm{~b}$ for the uncompensated and the FFE9 + DFE2 with ATC-SLICER and LMF respectively in $10 \mathrm{Gbps}$ NRZ. In Figure 4a with GVD only, the LMF is $1.5 \mathrm{~dB}$ better than the LMS for fixed thresholds, whereas with ATC-SLICER, it is $0.5 \mathrm{~dB}$ better than the LMS. 


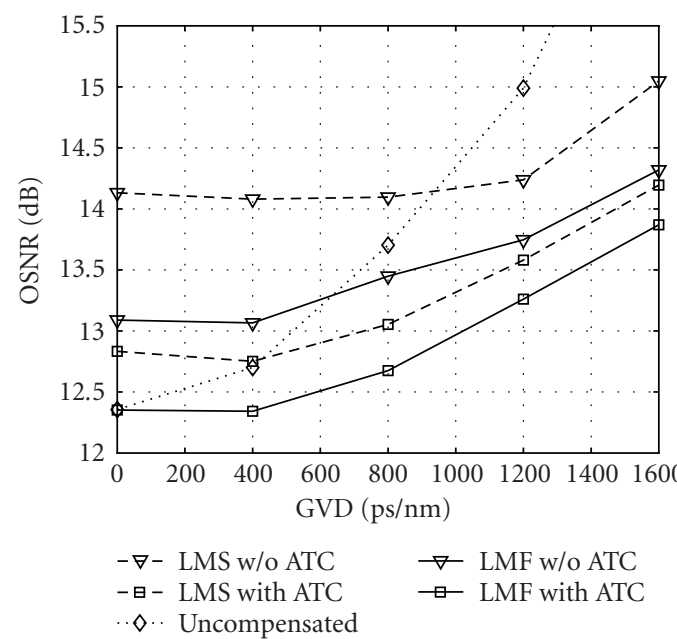

(a)

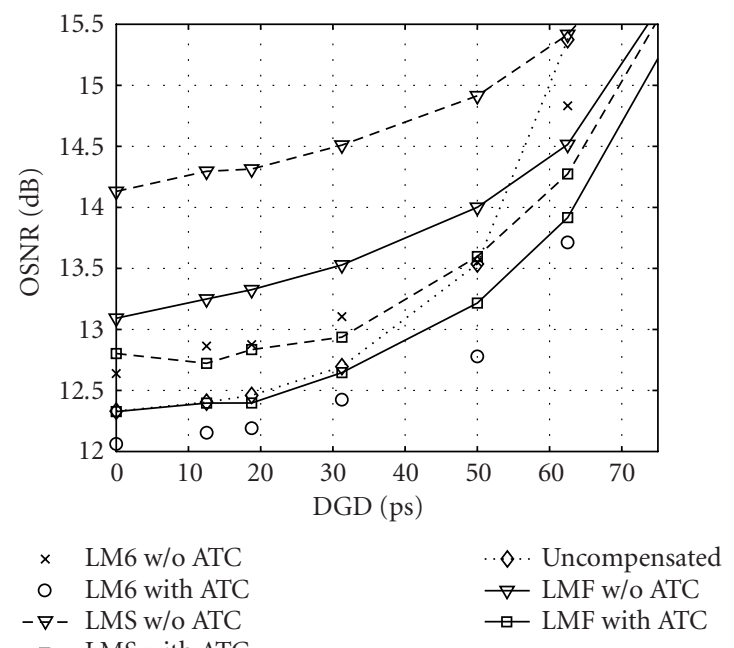

(b)
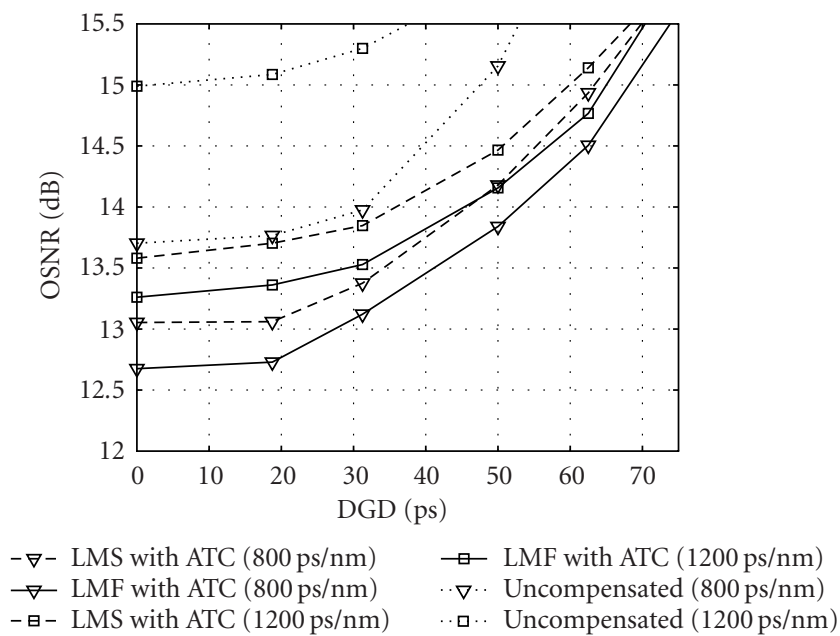

(c)

Figure 4: OSNR penalty when BER is set at $6 \times 10^{-5}$ (FEC-correctable error-rate level) for 10 Gbps NRZ under single and combined impairment conditions. The equalizer has a 9-tap fractionally spaced FFE and a 2-tap DFE with/without ATC-SLICER. (a) GVD only. (b) PMD only. (c) PMD combined with GVD (800 ps/nm).

It should be noted that a $3 \mathrm{~dB}$ gain means the possibility of doubling the transmission distance. In Figure $4 \mathrm{~b}$ with PMD only, the LMF and LM6 are 1.5- $2 \mathrm{~dB}$ better than the LMS for fixed thresholds, whereas the LM6 is almost $1 \mathrm{~dB}$ better than the LMS with ATC-SLICER. In addition, the LM6 with fixed thresholds has comparable performance to the LMS with optimized thresholds. In both cases, the LMF and LM6 with ATC-SLICER show less penalty than the uncompensated under no or mild dispersion condition while the LMS has higher penalty than the uncompensated. This implies that the LMS is potentially not penalty-free in a distortionfree environment, no matter how we optimize the threshold points. For the combined effect of PMD and GVD (800 or $1200 \mathrm{ps} / \mathrm{nm}$ ) depicted in Figure 4c, the LMF/LM6 are almost $0.5 \mathrm{~dB}$ better than the LMS with ATC. The trend indicates that the advantage of the LMN algorithms is more obvious in an environment of less distortion. Another implication is that the implementation of an ATC-SLICER can be simplified or avoided by employing the LMF or LM6 with minor increase in complexity of one or two multiplications as indicated in (1) and (2).

The advantage of the LMN algorithms over LMS is further illustrated in Figure 6, of which (a) and (b) demonstrate that the LMN can achieve much lower BER than the LMS. Note that the symbols such as "FFE9:0-3" in Figures 6a and $6 \mathrm{~b}$ mean the FFE equalizer type with 9 feedforward taps, no feedback taps, and the LM6 $(N=3)$. Figure $6 \mathrm{c}$ depicts the slicer input histograms for a 9-tap fractionally spaced FFE and shows how the LMN (LMF or LM8) can compact the distribution more effectively than the LMS. At DGD $=0$ picoseconds, LMS actually spreads the noise, causing lower BER and higher OSNR penalty, but the LMF maintains the noise variance.

Similar plots can be obtained for the $10 \mathrm{Gbps} \mathrm{RZ}$ case in Figure 7 where the performance of an FFE9 + DFE2 is shown to further demonstrate the advantage of the higher-order 

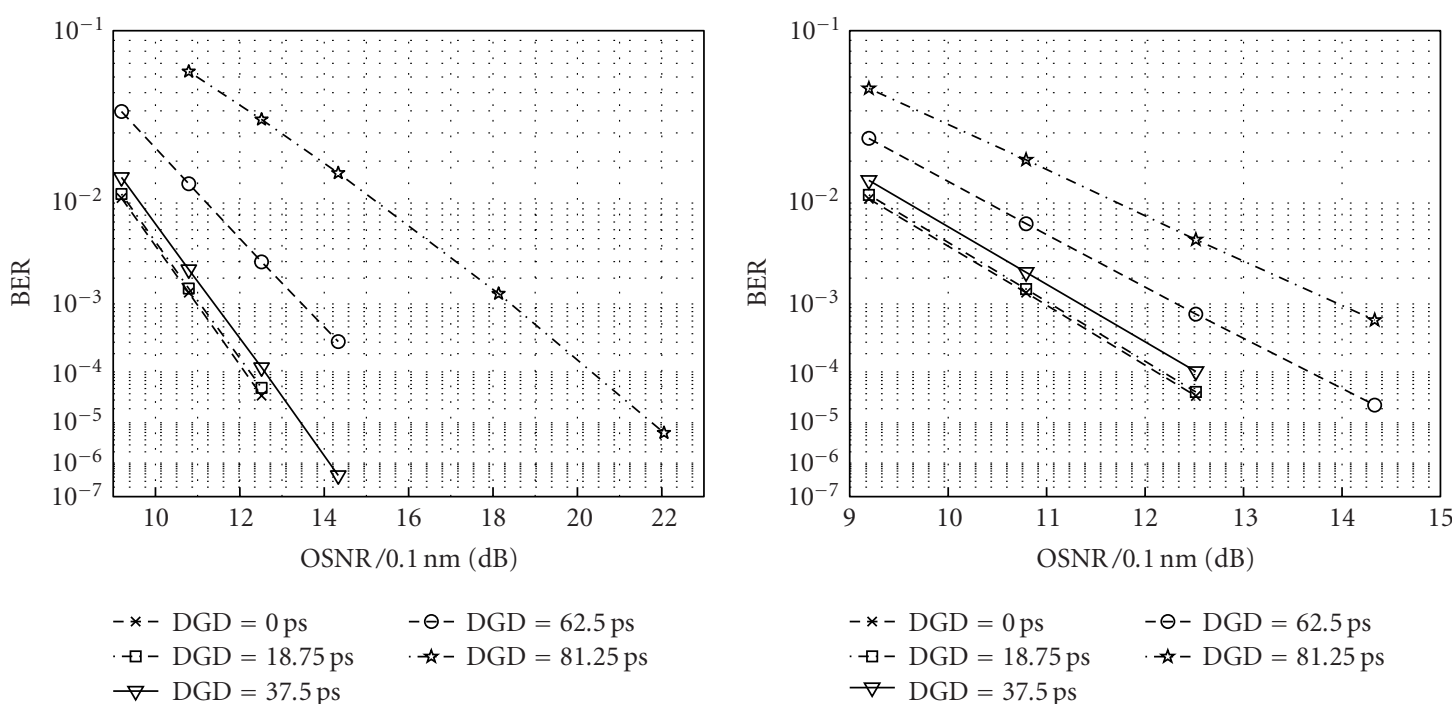

(a)

(b)

FIGURE 5: OSNR-BER plots with 1st-order PMD for NRZ format at 10 Gbps in Monte Carlo simulation. The equalizer has a 9-tap fractionally spaced FFE and a 2-tap DFE with ATC-SLICER optimized via the LMF. (a) Uncompensated with ATC. (b) FFE with DFE, ATC-SLICER, and LMF.
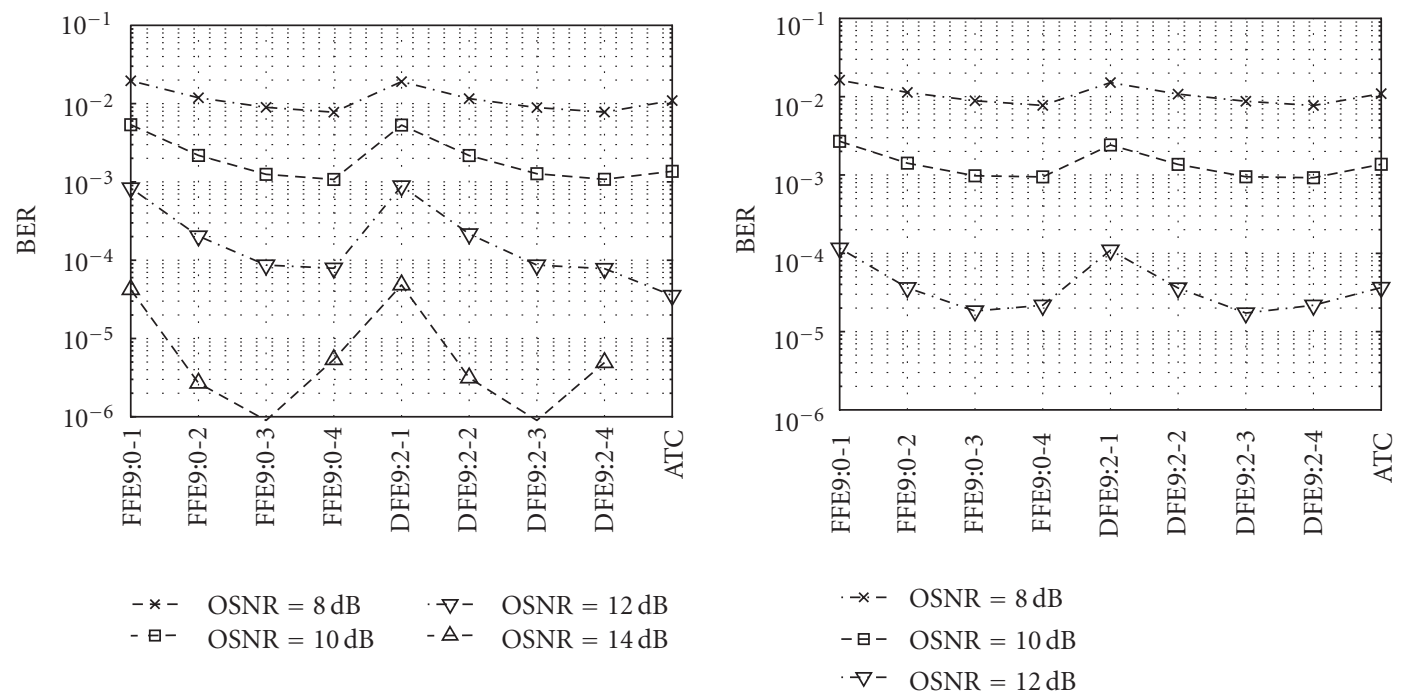

(a)

(b)
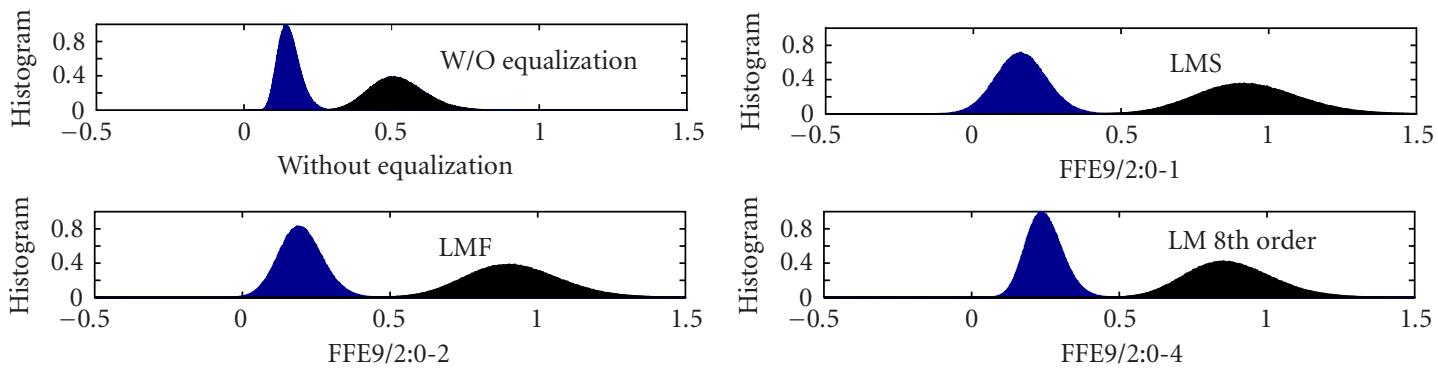

(c)

FIGURE 6: BER and slicer input histograms at DGD $=0$ picosecond of the first-order PMD for NRZ at 10 Gbps. The numbers in the equalizer type represent the following: number of FFE taps (NFFE), oversampling rate $(R>1$ : fractionally-spaced), number of DFE taps (NDFE), LMN order ( $N=1$ : LMS; $N=2$ : LMF; $N=3$ : LM6). (a) BER without ATC-SLICER. (b) BER with ATC-SLICER. (c) Slicer histogram for T/2-spaced 9-tap FFE. 

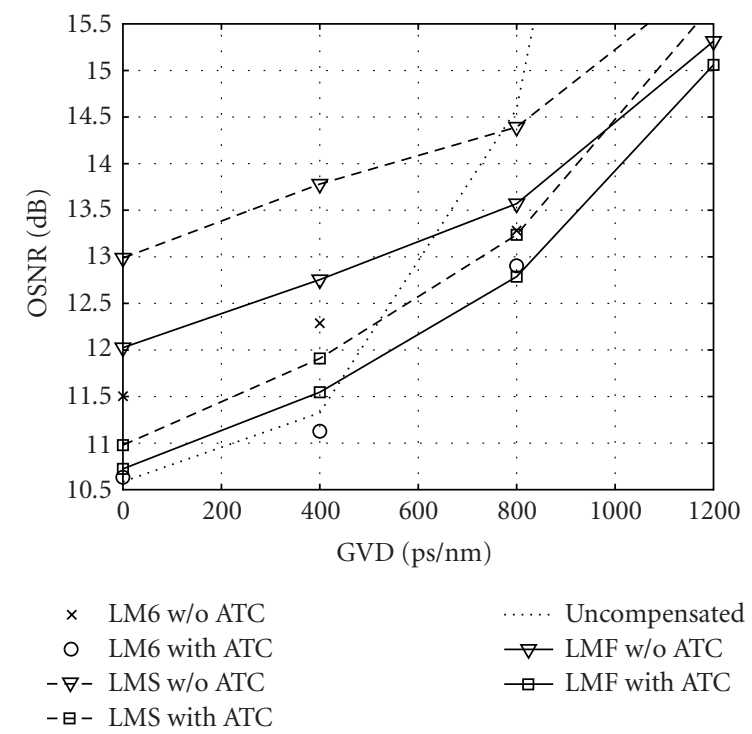

(a)

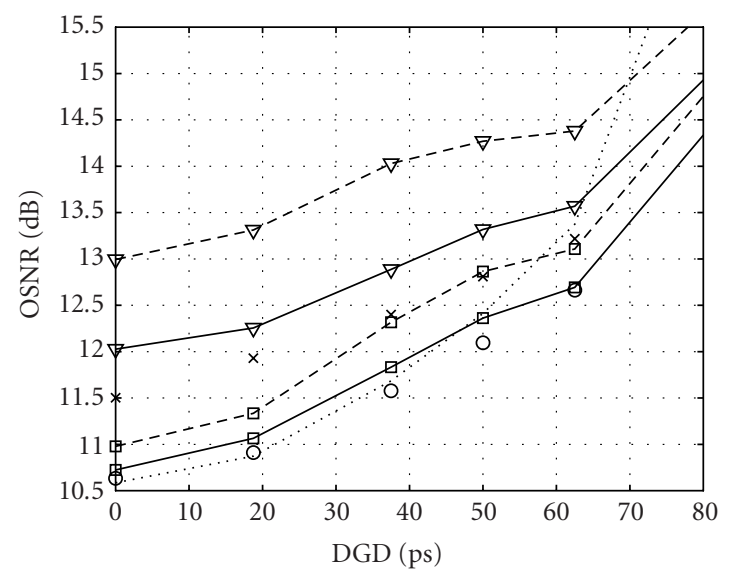

$\begin{array}{cl}\times & \text { LM6 w/o ATC } \\ \circ & \text { LM6 with ATC } \\ -\nabla- & \text { LMS w/o ATC } \\ - \text { - } & \text { LMS with ATC }\end{array}$

...... Uncompensated

$\nabla$ LMF w/o ATC

$\square$ LMF with ATC

(b)

FIGURE 7: OSNR penalty when BER is set at $6 \times 10^{-5}$ (FEC-correctable error-rate level) for 10 Gbps RZ under single and combined impairment conditions. (a) GVD only. (b) PMD only.

LMN algorithms over the conventional LMS for two different cases: only GVD and only PMD. With GVD only in Figure 7a, the LMN is $1.5 \mathrm{~dB}$ better without ATC-SLICER and $0.5 \mathrm{~dB}$ better with ATC-SLICER than the LMS. A similar trend can be found in Figure 7b affected by PMD alone.

\section{CONCLUSION}

LMS is well studied over the past several decades and considered optimal for a linear system with additive Gaussian noise. In a fiber-optic communication system, the dominant noise is optical noise (ASE). Due to the non-Gaussian nature of optical noise after nonlinear quadratic detection, the LMS is no longer optimal in ASE-dominant systems. The usual adaptive equalization techniques, so successful in electronic systems, cannot be directly applied to ASE-dominant fiber-optic systems in view of nonlinear square-law detection. In this paper, we demonstrate that its higher-order cousins in the LMN family are 1.5-2 dB better without ATC-SLICER and 0.5$1 \mathrm{~dB}$ better with ATC-SLICER than the LMS. In certain cases, the LMN with fixed thresholds even has comparable performance to the LMS with optimized thresholds. It suggests that the ATC-SLICER control unit may be eliminated in use of the LMN for the sake of less complexity. On the other hand, with a demanding performance criterion, the LMN with ATC-SLICER can be used to provide no back-to-back penalty (i.e. in absence of ISI-induced impairments). In comparison, the LMS is potentially penalized in a distortion-free environment, no matter how we optimize the threshold points, which is usually not acceptable in most receiver design goals.

\section{REFERENCES}

[1] P. P. Mitra and J. B. Stark, "The nonlinear limits to the information capacity of optical fibre communications," Nature, vol. 411, no. 28, pp. 1027-1030, 2001.
[2] S. Otte and W. Rosenkranz, "Electrical dispersion compensation for $10 \mathrm{~Gb} / \mathrm{s}$ transmission systems: simulation results," in Proc. 2nd Working Conference on Optical Network Design and Modelling (ONDEM '98), pp. 71-74, Rome, Italy, February 1998.

[3] H. Bulow, "Polarisation mode dispersion (PMD) sensitivity of a 10 Gbit/s transmission system," in Proc. European Conference on Optical Communication (ECOC '96), vol. 2, pp. 211-214, Oslo, Norway, September 1996.

[4] D. G. Messerschmitt, "Minimum MSE equalization of digital fiber optic systems," IEEE Trans. Commun., vol. 26, no. 7, pp. 1110-1118, 1978.

[5] E. Desurvire, Erbium-Doped Fiber Amplifiers: Principles and Applications, Wiley-Interscience, New York, NY, USA, 1994.

[6] R. Hopper, "Optimum equalisers for binary digital transmission systems and their application to optical receivers," IEE Proceedings J-Optoelectronics, vol. 136, no. 2, pp. 137-140, 1989.

[7] J. H. Winters and R. D. Gitlin, "Electrical signal processing techniques in long-haul fiber-optic systems," IEEE Trans. Commun., vol. 38, no. 9, pp. 1439-1453, 1990.

[8] J. H. Winters, "Equalization in coherent lightwave systems using a fractionally spaced equalizer," J. Lightwave Technol., vol. 8, no. 10, pp. 1487-1491, 1990.

[9] S. Kasturia and J. H. Winters, "Techniques for high-speed implementation of nonlinear cancellation," IEEE J. Select. Areas Commun., vol. 9, no. 5, pp. 711-717, 1991.

[10] N. Swenson, B. Shoop, and J. Cioffi, "Precluding nonlinear ISI in direct detection long-haul fiber optic systems," IEEE Photon. Technol. Lett., vol. 3, no. 2, pp. 182-184, 1991.

[11] J. Cartledge, R. McKay, and M. Nowell, "Performance of smart lightwave receivers with linear equalization," J. Lightwave Technol., vol. 10, no. 8, pp. 1105-1109, 1992.

[12] H. Bulow, D. Schlump, J. Weber, B. Wedding, and R. Heidemann, "Electronic equalization of fiber PMD-induced distortion at 10 Gbit/s," in Optical Fiber Communication Conference and Exhibit (OFC'98), pp. 151-152, San Jose, Calif, USA, February 1998. 
[13] F. Cariali, F. Martini, P. Chiappa, and R. Ballentin, "Electronic compensation of PMD and chromatic dispersion with an IC in 10 Gbit/s transmission system," Electronics Letters, vol. 36, no. 10 , pp. $889-891,2000$.

[14] H. Bulow, F. Buchali, W. Baumert, R. Ballentin, and T. Wehren, "PMD mitigation at $10 \mathrm{Gbit} / \mathrm{s}$ using linear and nonlinear integrated electronic equaliser circuits," Electronics Letters, vol. 36, no. 2, pp. 163-164, 2000.

[15] F. Buchali, H. Bulow, and W. Kuebart, "Adaptive decision feedback equalizer for $10 \mathrm{Gbit} / \mathrm{s}$ dispersion mitigation," in Proc. European Conference on Optical Communication (ECOC '00), Munich, Germany, September 2000, p. 5.2.5.

[16] H. Bulow and G. Thielecke, "Electronic PMD mitigationfrom linear equalization to maximum-likelihood detection," in Optical Fiber Communication Conference and Exhibit (OFC '01), vol. 3, pp. WAA3-1-WAA3-3, Anaheim, Calif, USA, March 2001.

[17] H. Bulow, "PMD mitigation by optic and electronic signal processing," in Proc. IEEE Lasers and Electro-Optics Society (LEOS '01), vol. 2, pp. 602-603, San Diego, Calif, USA, November 2001.

[18] M. Win, J. H. Winters, and G. Vitetta, Equalization Techniques for Mitigating Transmission Impairments, chapter 18, Academic Press, New York, NY, USA, 2002.

[19] T. Adali, "Applications of signal processing to optical fiber communications," in Proc. IEEE Lasers and Electro-Optics Society (LEOS '02), vol. 2, pp. 623-624, Glasgow, UK, November 2002.

[20] L. Moller, A. Thiede, S. Chandrasekhar, et al., "ISI mitigation using decision feedback loop demonstrated with PMD distorted 10 Gbit/s signals," Electronics Letters, vol. 35, no. 24, pp. 2092-2093, 1999.

[21] H. Bulow, R. Ballentin, W. Baumert, F. Buchali, and W. Kuebart, "Adaptive PMD mitigation at $10 \mathrm{Gbit} / \mathrm{s}$ using an electronic SiGe equaliser IC," in Proc. European Conference on Optical Communication (ECOC '99), pp. 138-139, Nice, France, September 1999.

[22] H. F. Haunstein, K. Sticht, A. Dittrich, M. Lorang, W. SauerGreff, and R. Urbansky, "Implementation of near optimum electrical equalization at $10 \mathrm{Gbit} / \mathrm{s}$," in Proc. European Conference on Optical Communication (ECOC '00), vol. 3, pp. 223 224, Munich, Germany, September 2000.

[23] H. F. Haunstein, K. Sticht, A. Dittrich, W. Sauer-Greff, and R. Urbansky, "Design of near optimum electrical equalizers for optical transmission in the presence of PMD," in Optical Fiber Communication Conference and Exhibit (OFC '01), vol. 3, pp. WAA4-1-WAA4-3, Anaheim, Calif, USA, March 2001.

[24] K. Shimoda, G. Takahashi, and C. H. Townes, "Fluctuations in amplification of quanta with application to maser amplifiers," Journal of the Physical Society of Japan, vol. 12, no. 6, pp. 686700, 1957.

[25] J. Perina, "Superposition of coherent and incoherent fields," Physics Letters A, vol. 24, no. 6, pp. 333-334, 1967.

[26] H. A. Haus, Electromagnetic Noise and Quantum Optical Measurements, Springer-Verlag, New York, NY, USA, 2000.

[27] L. Kazovsky, S. Benedetto, and A. Willner, Optical Fiber Communication Systems, Artech House, Norwood, Mass, USA, 1996.

[28] U.-V. Koc, "Noise property and optimum electronic PMD compensation for strongly/weakly optically filtered differential phase shift keying (DPSK) modulation," in Optical Fiber Communication Conference and Exhibit (OFC '03), vol. 2, pp. 596-599, Atlanta, Ga, USA, March 2003.

[29] H. Bulow, "Electronic equalization of transmission impairments," in Optical Fiber Communication Conference and Exhibit (OFC'02), pp. 24-25, Anaheim, Calif, USA, March 2002.
[30] G. L. Frazer, M. W. Goodwin, K. E. Leonard, J. P. Moffatt, and F. Zhang, "Static and dynamic performance of an adaptive receiver for 10 Gbps optical transmission," in Proc. European Conference on Optical Communication (ECOC '00), vol. 2, pp. 113-114, Munich, Germany, September 2000.

[31] H. Bulow, W. Baumert, F. Buchali, and W. Kuebart, "Adaptation of an electronic PMD mitigator by maximization of the eye opening," in Proc. European Conference on Optical Communication (ECOC '00), Munich, Germany, September 2000, paper P3.10.

[32] M. Fregolent, S. Herbst, H. Soehnle, and B. Wedding, "Adaptive optical receiver for performance monitoring and electronic mitigation of transmission impairments," in Proc. European Conference on Optical Communication (ECOC '00), Munich, Germany, September 2000, paper 2.1.2.

[33] K. Sticht, H. F. Haunstein, M. Lorang, W. Sauer-Greff, and R. Urbansky, "Adaptation of electronic PMD equalizer based on BER estimation derived from FEC decoder," in Proc. 27th European Conference on Optical Communication (ECOC '01), pp. 454-455, 2001.

[34] E. Walach and B. Widrow, "The least mean fourth (LMF) adaptive algorithm and its family," IEEE Trans. Inform. Theory, vol. 30, no. 2, pp. 275-283, 1984.

[35] C. L. Nikias and A. P. Petropulu, Higher-Order Spectra Analysis-A Nonlinear Signal Processing Framework, PTR Prentice Hall, Englewood Cliffs, NJ, USA, 1993.

[36] U.-V. Koc, K. Tu, and N. Kaneda, "Adaptive electronic equalization using higher-order statistics for PMD compensation in long-haul fiber-optic systems," in European Conference on Optical Communication (ECOC'02), Copenhagen, Denmark, September 2002, paper 7.1.5.

[37] R. D. Gitlin, J. F. Hayes, and S. B. Weinstein, Data Communications Principles, Plenum Press, New York, NY, USA, 1992.

[38] B. Hassibi, A. H. Sayed, and T. Kailath, " $H^{\infty}$ optimality of the LMS algorithm," IEEE Trans. Signal Processing, vol. 44, no. 2, pp. 267-280, 1996.

[39] N. Kaneda, E. C. Burrows III, B. Le, K. Sticht, M. Hartlaub, and R. M. Jopson, "Polarization mode dispersion tolerance of an adaptive threshold receiver", in LEOS Topical Meeting, pp. TuN3-39-TuN3-40, 2002.

[40] M. Kawai, H. Watanabe, T. Ohtsuka, and K. Yamaguchi, "Smart optical receiver with automatic decision threshold setting and retiming phase alignment," J. Lightwave Technol., vol. 7, no. 11, pp. 1634-1640, 1989.

Ut-Va Koc received the Ph.D. degree from the University of Maryland, College Park, and the B.S. degree from National Chiao Tung University. He is currently a Distinguished Member of Technical Staff (DMTS) at High Speed Electronics Research, Bell Labs, Lucent Technologies, Murray Hill, New Jersey. He has published one book, a number of peer-reviewed papers, and book chapters on signal processing in communications and multimedia, in addition to 5 patents granted or in submission. He has been active in serving as a reviewer for various journals and conferences, Editor for EURASIP Journal on Applied Signal Processing (JASP), Guest Editor for a special issue in JASP, and Guest Cochair in various international conferences. His current research interests include electronic and optical signal processing for optical/electronic wireline/wireless communication, analog/mixed signal processing for high-speed data conversion, and multimedia signal processing. 